\title{
Effects of chlorpyrifos-methyl, chlormequat, deltamethrin, glyphosate, pirimiphos-methyl, tebuconazole and their mixture on oxidative stress and toxicity in HUVEC cell line
}

\author{
Çiğgem Sevim ${ }^{10}$, Ali Taghizadehghalehjoughi² (D), Mehtap Kara³ (1) \\ 'Kastamonu University, Faculty of Medicine, , Deparment of Medical Pharmacology, Kastamonu, Turkey \\ ${ }^{2}$ Ataturk University, Faculty of Veterinary, Deparment of Pharmacology and Toxicology, Erzurum, Turkey \\ ${ }^{3}$ Istanbul University, Faculty of Pharmacy, Deparment of Pharmaceutical Toxicology, Istanbul, Turkey
}

ORCID IDs of the authors: C..S. 0000-0002-0575-3090; A.T. 0000-0002-3506-0324; M.K. 0000-0001-7764-5593

Cite this article as: Sevim, C., Taghizadehghalehjoughi, A., \& Kara, M. (2021). Effects of chlorpyrifos-methyl, chlormequat, deltamethrin, glyphosate, pirimiphos-methyl, tebuconazole and their mixture on oxidative stress and toxicity in HUVEC cell line. istanbul Journal of Pharmacy, 51(2), 183-190.

\begin{abstract}
Background and Aims: Humans and animals have daily contact with various chemicals, including food additives, pesticides, antibiotics, other veterinary drugs, and other xenobiotics. Pesticide exposure causes many health disorders. Mixed exposure to pesticides is an important issue for human and environmental health.

Methods: In this study, we have determined the cytotoxicity of chlormequat pirimiphos-methyl, glyphosate, tebuconazole, chlorpyrifos-methyl, deltamethrin, and the mixture of these six pesticides. We further investigated the role of oxidative stress, total oxidant status (TOS), lactate dehydrogenase (LDH) and antioxidant defense mechanism TOS, total glutathione (GSH) levels with the observed cytotoxicity.

Results: In this study, the mixtures of pesticides reduced total antioxidant status (TAS) and GSH level one by one and increased the reactive oxygen species (ROS) generation in HUVECs, respectively. The results also showed a significant contribution of oxidative stress on cytotoxicity during pesticide mixture exposure.

Conclusion: The findings are that pesticide mixture exposure might have an impact on human health risk at contaminated sites and under occupational exposure conditions.

Keywords: Chlorpyrifos-methyl, chlormequat, deltamethrin, glyphosate, pirimiphos-methyl, tebuconazole, oxidative stress, HUVECS
\end{abstract}

\section{INTRODUCTION}

Pesticides are commonly used compounds in farming that have a wide range of classes, such as insecticides, herbicides, fungicides, nematicides, acaricides, rodenticides, avicides, wood preservatives, and antifoulants. A remarkable amount of these pesticides spreads into the environment and this brings about immunotoxicity, carcinogenesis, and endocrine and developmental toxicity. Authorities such EFSA, EPA, and the Food, Drug, and Cosmetic Act, attaches importance to assessing pesticide compound mixture effects on the environment and living organisms (Laetz, Baldwin \& Collier., 2009; Oyesola, Iranloye \& Adegoke, 2019). Risk assessment of pesticide mixture is a highly important issue due to environmental and human exposure. People are always exposed to pesticide combinations due to protective strategies against pests in farms in farming. However, there is still no strict rule for risk assessment of pesticide compound mixture. Different combination risk assessment models 
can be seen in the literature. The most common risk assessment model is the dose addition model found in the European Food Safety Authority (EFSA) guidelines (Staal et al., 2018).

Conazole fungicides are widely used for agriculture and also treatment of mycosis and candida infections in humans and animals. Conazole fungicides cause disarrangement of fungal membranes via inhibition of cytochrome P450 enzyme lanosterol 14a-demethylase (CYP51). CYP51 is one of the main elements of ergosterol biosynthesis and normal fungal membrane integrity. Canozole fungicides have side effects such as endocrine disruption. These compounds exert their endocrine disrupting effects via CYP51 and CYP19 inhibition, which play a significant role as steroidogenic enzymes (Roelofs, Temming, Piersma, van der Beg \& van Duursen, 2014). Tebuconazole is a widely used fungicide that has a sterol demethylation inhibitor effect. It has been reported that the Tebuconazole plasma half-life is approximately 600 days, and it causes toxic effects on the thyroid, liver, nervous system, and reproductive organs. In addition, it also exposes developmental and genetic toxicity (Li et al., 2020).

Chlormequat chloride is a quaternary ammonium compound which is used for floriculture as a plant growth regulator by reducing longitudinal shoot growth effect (Vijitharan, Warnasekare, Lokunarangoda, Farah \& Siribaddana, 2016). Exposure to human beings is quite common. According to $\mathrm{WHO}$, chlormequat is excreted $98 \%$ invariably and its acceptable daily intake (ADI) level is $0.05 \mathrm{mg} / \mathrm{kg}$.bw. It is totally eliminated from the body within $46 \mathrm{~h}$. It is important to investigate the potential toxic effects of chlormequat on cellular mechanisms due to the wide range of species that are exposed to it (Xiagedeer, Wu, Liu, \& Hao, 2016). Organophosphorus pesticides (OP) are the most common pesticide type used worldwide. OPs are absorbed via inhalation, skin, eyes, and ingestion. The main acute toxic effect is inhibition of the acetylcholinesterase enzyme, which results in a cholinergic crisis. Pirimiphos-methyl belongs to the OP group of pesticides, which are non-cumulative and broad spectrum compounds. Even though pirimiphos-methyl is classified as "no reprotoxin, no teratogen" by OECD, its harmful effects on development and on the reproductive and immune systems have been shown in studies (Oyesola et al. 2019; Olsvik, Berntssen \& Søfteland, 2017; Anogwih, 2014). Glyphosate is a non - selective organophosphorus herbicide and it has bioaccumulation potential in the environment. Glyphosate exposure causes toxic effects in mammalians by increasing oxidative stress levels. Due to its common usage, this pesticide residue can be found in food and environmental samples. The main toxicity mechanism of glyphosate increases the emergence of ROS and causes disruption on cellular macromolecules by ROS (Cai et al., 2020; Odetti et al., 2020; Zhang, Yang, Ma, Shi \& Chen, 2020). Chlorpyrifos is another widely used broad-spectrum organophosphorus insecticide. It has been reported that chlorpyrifos might have some toxic effects on organs and systems. Its common toxic effect is on the nervous system by acetylcholinesterase (AChE) inhibition. Its main toxic effect mechanism is associated with cellular oxidative stress increment (Deng, Zhang, Lu, Zhao \& Ren, 2016; Dokuyucu, et al., 2016). Deltamethrin is part of the potent pyrethroid class of insecticides and acaricides. Also, deltamethrin has been used for controlling human diseases caused by mosquitos that are vectors of Zika virus and Dengue virus. It has been reported that deltamethrin exerts its toxic effects by increasing cellular ROS and reactive nitrogen species (RNS). Oxidative stress remarkably increases the deltamethrin toxicity mechanism (Lu et al., 2019).

Oxidative stress is a cellular imbalance condition which is formed between free radical production and the antioxidant defense system. Several chemicals may cause free radical production and through this, these radicals interact with important cellular molecules and impair their functions pathologically (Abdollahi, Ranjbar, Shadnia, Nikfar \& Rezaie, 2004). Pesticide exposure may cause reactive oxygen species production in the cell that exceeds antioxidant defenses system. The cellular antioxidant system includes several molecules and enzymes, including reduced glutathione (GSH), non-protein thiols, catalase (CAT), glutathione-S-transferases (GST), superoxide dismutase (SOD), and glutathione reductase (GR) (Ferreira, et al., 2010). The pesticide-induced oxidative stress molecular mechanism has still not been clarified and is under scrutiny by scientists seeking to evaluate risk factors and understand related pathologic diseases (Agrawal \& Sharma, 2010).

There are limited studies in the literature for these six pesticides' effects on cellular oxidative stress in endothelial cells. HUVECs are cell models that are frequently used to investigate the underlying mechanisms of endothelial diseases (MedinaLeyte, Domínguez-Pérez, Mercado, Villarreal-Molina, \& JacoboAlbavera, 2020) In this study, we have evaluated the toxicity of singly and mixture In this study, we have evaluated the toxicity of chlorpyrifos-methyl, chlormequat, deltamethrin, glyphosate, pirimiphos-methyl, tebuconazole singly and as a mixture. Further oxidative stress-inducing potential was evaluated in HUVECs. This is the first study of the effects of this pesticide mixture on HUVECs.

\section{MATERIALS AND METHODS}

\section{Cell culture and cell viability assays}

The Human Umbilical Vein Endothelial (HUVEC) cell line was provided from American Type Culture Collection (ATCC ${ }^{\circledast}$ PCS100-010 ${ }^{\mathrm{TM}}$ ). Cells were grown in RPMI 1640 (GIBCO, Uxbridge, UK) including 10\% fetal bovine serum (GIBCO, Uxbridge, UK), $100 \mathrm{U} / \mathrm{ml}$ penicillin, and $100 \mu \mathrm{g} / \mathrm{ml}$ streptomycin (GIBCO, Uxbridge, UK), with incubation conditions of $37^{\circ} \mathrm{C}$ and a humidified atmosphere with $5 \% \mathrm{CO}_{2}$. Cell passage was performed with $0.025 \%$ trypsin- $0.02 \%$ EDTA after cell co-fluency. Cells passage was performed twice a week. HUVEC cells are a sufficient model for investigating the molecular mechanisms of cardiovascular diseases under different chemical exposure conditions (Medina-Leyte et al., 2020).

Chlormequat chloride, pirimiphos-pethyl, glyphosate, tebuconazole, chlorpyrifos-methyl, deltamethrin, and their mixture's effects on the HUVECs viability were assessed by MTT test, which depends on measuring the reduction of 3-(4,5-dimethylthiazol-2-yl)-2,5-diphenyltetrazolium bromide (MTT) compound to formazan by the mitochondrial enzyme succinate dehydrogenase. After a $24 \mathrm{~h}$ exposure duration, cells were incubated with MTT for $3 \mathrm{~h}$ at $37^{\circ} \mathrm{C}$ in a humidified atmosphere (95\%) with $5 \% \mathrm{CO}_{2}$. After incubation, cell media was discarded, 
$100 \mu \mathrm{L}$ DMSO was added into each well, and absorbance of formazan compound was measured with spectrophotometry (Epoch, Biotek) at a wavelength of $595 \mathrm{~nm}$. Pesticide exposure concentrations are shown in Table 1 (Roelofs et al. 2014).

Table 1. Pesticide exposure concentrations for cell viability assay.

\begin{tabular}{|lc|}
\hline Pesticide & Concentrations (mM) \\
\hline Chlormequat chloride & $25,50,100$ and 200 \\
Pirimiphos-Methyl & $25,50,100$ and 200 \\
Glyphosate & $25,50,100$ and 200 \\
Potassium & \\
Tebuconazole & $25,50,100$ and 200 \\
Chlorpyrifos-methyl & $25,50,100$ and 200 \\
Deltamethrin & $2.5,5,10$ and 20 \\
Mix & 50 and 200 \\
\hline
\end{tabular}

\section{Oxidative stress quantification assays}

Lactat Dehydrogenase (LDH) Assay: LDH assay was performed with an LDH assay kit (ab102526-abcam). This assay was based on quantifying LDH activity via reducing NAD to NADH, then $\mathrm{NADH}$ interacts with a specific kit reagent that produces a color which can be detected at $450 \mathrm{~nm}$. Cell lysates were mixed with reaction reagent and the colored sample was read at 450 $\mathrm{nm}$ with a spectrophotometer (Epoch, Biotek).

Total Antioxidant Status (TAS): TAS assay was performed via a TAS reagent kit (Rel Assay Diagnostics - Turkey) through the manufacturer's service bulletin. This assay was based on antioxidants reducing kit ABST component, and the absorbance change was detected by spectrophotometer at $660 \mathrm{~nm}$. The assay is calibrated via trolox equivalent. Cell lysates and standards were mixed with reagent 1 and this mixture was measured spectrophotometrically. After reagent 2 was added into the mixture, the measurement was done at 5 min interval at $660 \mathrm{~nm}$.
Total Oxidant Status (TOS): TOS assay was performed by TOS a Reagent kit (Rel Assay Diagnostics - Turkey) with the manufacturer's instruction. This assay was based on oxidants in the sample which oxidize the ferrous ions. Oxidized ferric ions make a colored complex that can be measured by spectrophotometer at $530 \mathrm{~nm}$. The assay is calibrated via hydrogen peroxide. Cell lysates and standards were mixed with reagent 1 and the first spectrophotometric measurement wasdone with $530 \mathrm{~nm}$. Then reagent 2 was added into the mixture and absorbance measurement was was done at 5 minutes interval.

Total Glutathione (GSH) Assay: GSH assay was performed by Glutathione Detection Assay Kit (Fluorometric) (Abcam-ab65322). This kit detects both reduced and oxidized glutathione. This assay is based on monochlorobimane (MCB), which is adducted with GSH that is catalyzed by GST. Bound MCB creates fluorescent blue light $(E x / E m=380 / 461 \mathrm{~nm})$, which is detected by fluorometer (CARY ECLIPSE - USA).

\section{RESULTS}

\section{Cell viability}

According to MTT assay results, $I C_{50}$ values of five pesticides and their combination exposure effects have been listed in Table 2 (Figure 1). 50 and $200 \mathrm{mM}$ concentrations of each pesti-

\section{Table 2. IC $\mathrm{C}_{50}$ values of pesticides according to MTT} results.

\begin{tabular}{|lc|}
\hline Pesticide & IC $_{50}$ value $(\mathrm{mM})$ \\
\hline Chlorpyrifos-methyl & 51.836 \\
Pirimiphos-Methyl & 52.729 \\
Glyphosate & 151.198 \\
Chlormequat Chloride & 54.232 \\
Tebuconazole & 25.837 \\
Deltamethrin & 8.567 \\
Mix 50 and mix 200 & 38.060 \\
\hline
\end{tabular}
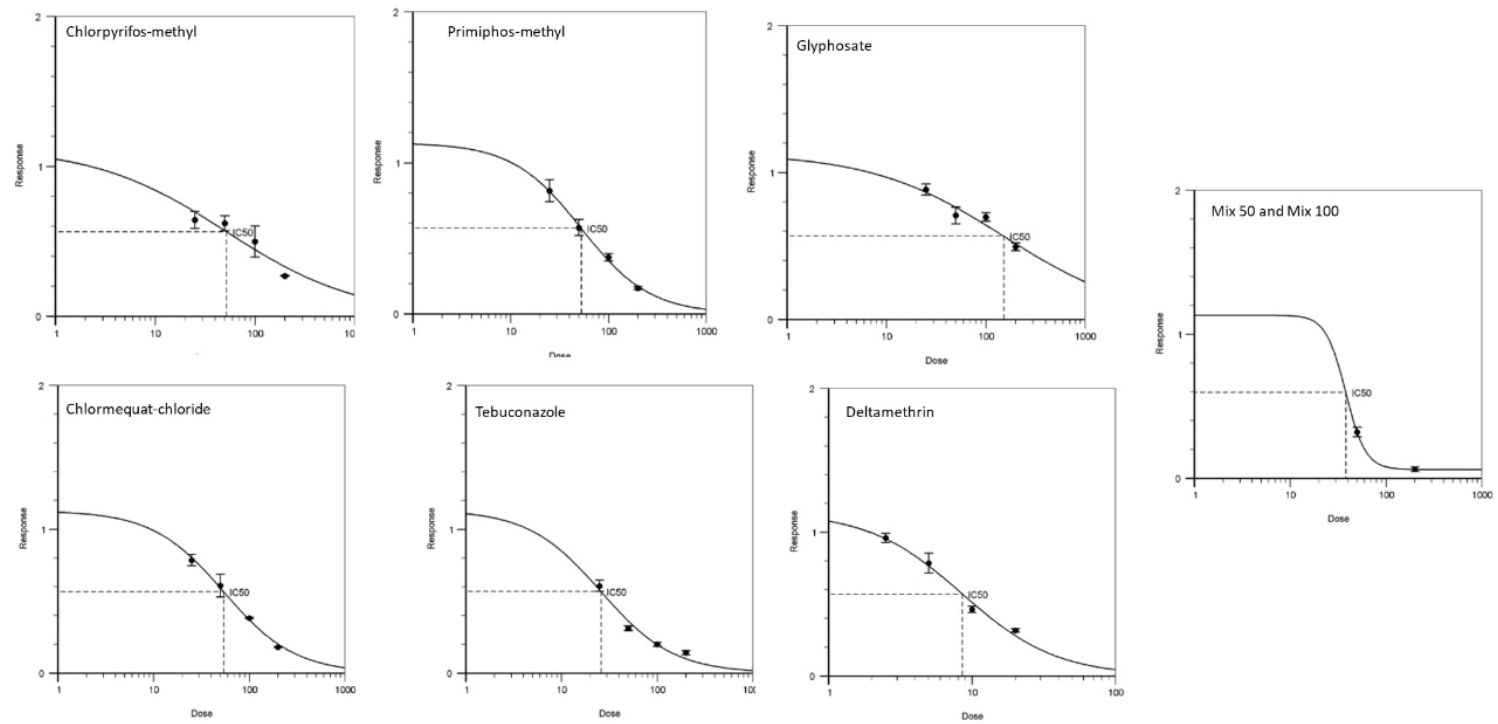

Figure 1. Cell viability assay graphs of six pesticided and their combination at 50 and $100 \mathrm{mM}$ concentrations. $I C_{50}$ values of each pesticide were calculated via graphs slope formulation. 
cide were chosen for combination exposure. For LDH, TAS, TOS and GSH assays 25, 50, 100 and 200 mM concentrations were chosen for $24 \mathrm{~h}$ exposure. We used concentrations that were above the $I C_{50}$ values to compare higher concentration effects on oxidative stress parameters.

\section{LDH assay}

$\mathrm{LDH}$ values significantly decreased at $25 \mathrm{mM}$ concentration for all six pesticides compared to the control group. Chlormequat chloride exposure to all experimental concentrations decreased LDH level. Increased LDH levels were observed in higher concentrations of chlorpyrifos-methylp and pirimiphos-methyl groups. 50 and 200 mM mix groups showed a significant decrease in LDH levels compared to the control group (Figure 2). LDH levels after pesticide exposure are shown in Table 3.

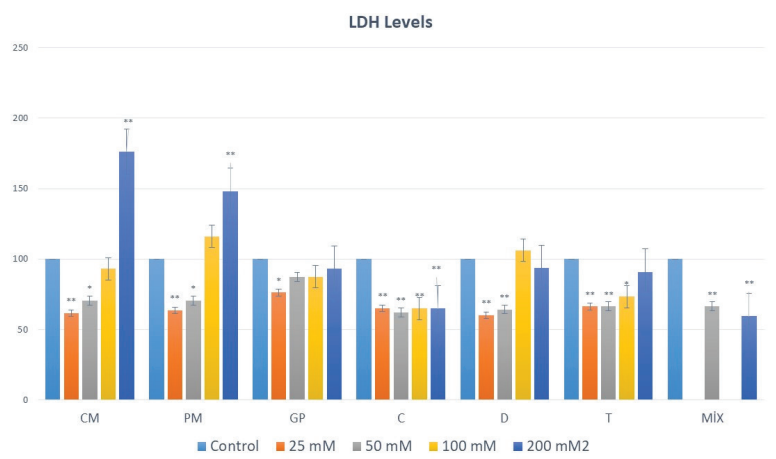

Figure 2. $\mathrm{LDH}$ assay results of six pesticides. ${ }^{*} \mathrm{p}<0,05 ;{ }^{*} \mathrm{p}<0,001$. CM: Chlorpyrifos-methyl, PM: Pirimiphos-Methyl, GP: Glyphosate, C: Chlormequat Chloride, D: Deltamethrin, T: Tebuconazole.

\section{Total antioxidant status}

TAS levels decreased in concentration dependent, but not significantly in the chlorpyrifos-methyl group. TAS levels decreased in pthe pirimiphos-methyl and deltamethrin groups. TAS levels decreased in the glyphosate, chlormequat chloride, and tebuconazole groups only in 25 and $200 \mathrm{mM}$ exposure concentrations compared to the control group. TAS levels decreased in mixed groups compared to the control group (Figure 3). TAS levels after pesticide exposure are shown in Table 3.

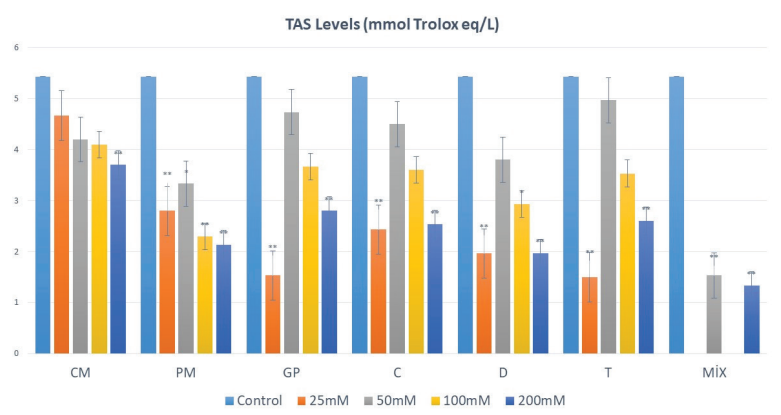

Figure 3. TAS assay results of six pesticides. ${ }^{*} p<0,05 ;{ }^{* *} p<0,001$. CM: Chlorpyrifos-methyl, PM: Pirimiphos-Methyl, GP: Glyphosate, C: Chlormequat Chloride, D: Deltamethrin, T: Tebuconazole.

\section{Total oxidant status}

TOS levels increased significantly in pthe pirimiphos-m, glyphosate, chlormequat chloride, deltamethrin, and tebuconazole single exposures and in the mixture exposure compared to the control group (Figure 4). TOS levels after pesticide exposure are shown in Table 3.

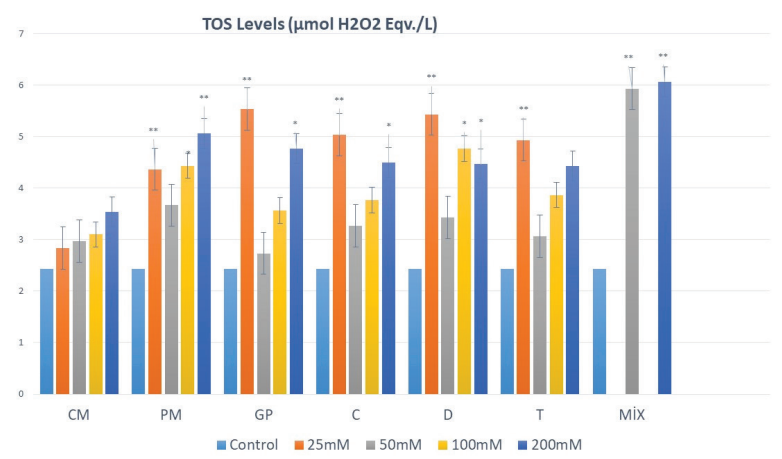

Figure 4. TOS assay results of six pesticides. ${ }^{*} p<0,05 ;{ }^{* *} p<0,001$. CM: Chlorpyrifos-methyl, PM: Pirimiphos-Methyl, GP: Glyphosate, C: Chlormequat Chloride, D: Deltamethrin, T: Tebuconazole.

\section{Glutathione levels}

GSH levels decreased in all exposure concentrations significantly in the chlormequat chloride, deltamethrin, tebuconazole, and mix groups. In the glyphosate group 50, 100, and 200 mM concentrations, GSH levels decreased significantly compared to the control group. In pthe Pirimiphos-methyl 100 and 200 mM concentrations, GSH levels decreased significantly compared to the control group. However, for the $25 \mathrm{mM}$ group, GSH level increased dramatically. For the chlorpyrifosmethyl group 100 and 200 mM concentrations, GSH levels decreased significantly compared to the control group (Figure 5). GSH levels after pesticide exposure are shown in Table 3.

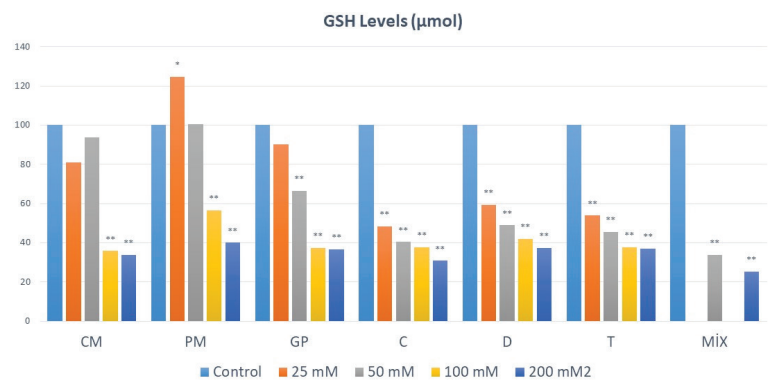

Figure 5. GSH assay results of six pesticides. ${ }^{*} p<0,05 ;{ }^{* *} p<0,001$. CM: Chlorpyrifos-methyl, PM: Pirimiphos-Methyl, GP: Glyphosate, C: Chlormequat Chloride, D: Deltamethrin, T: Tebuconazole.

\section{DISCUSSION}

Pesticides are commonly used chemicals that protect agricultural products from weeds, insects, fungus, and rodents. However, pesticides are mostly toxic for the environment and living organisms. Also, pesticides are commonly used for controlling malaria and dengue disease vectors and controlling plant growth in public places, such as parks and gardens, 
Table 3. LDH, TASS, TOS and GSH levels in HUVEC cells after $24 \mathrm{~h}$ pesticide exposure duration.

\begin{tabular}{|c|c|c|c|c|}
\hline Pesticides & LDH (\%) & $\begin{array}{c}\text { TAS } \\
\text { (mmolTrolox eq/L) }\end{array}$ & $\begin{array}{c}\text { TOS } \\
\left(\mu \mathrm{mol} \mathrm{H}_{2} \mathrm{O}_{2} \text { eq/L) }\right.\end{array}$ & $\begin{array}{c}\text { GSH } \\
\text { (\% for control group) }\end{array}$ \\
\hline Control & 100 & $5.4 \pm 0.1$ & $2.4 \pm 0.1$ & 100 \\
\hline \multicolumn{5}{|c|}{ Chlormequat chloride (mM) } \\
\hline 25 & 65.1 & $2.4 \pm 0.1$ & $5.1 \pm 0.2$ & 48.4 \\
\hline 50 & 62.2 & $4.5 \pm 0.2$ & $3.2 \pm 0.2$ & 40.3 \\
\hline 100 & 65.1 & $3.6 \pm 0.2$ & $3.7 \pm 0.1$ & 37.6 \\
\hline 200 & 61 & $2.5 \pm 0.1$ & $4.5 \pm 0.1$ & 30.7 \\
\hline \multicolumn{5}{|c|}{ Pirimiphos-Methyl (mM) } \\
\hline 25 & 63.6 & $2.8 \pm 0.1$ & $4.3 \pm 0.1$ & 100.5 \\
\hline 50 & 70.6 & $3.3 \pm 0.2$ & $3.6 \pm 0.1$ & 100.3 \\
\hline 100 & 116.8 & $2.3 \pm 0.2$ & $4.4 \pm 0.05$ & 56.6 \\
\hline 200 & 140.1 & $2.1 \pm 0.3$ & $5.1 \pm 0.2$ & 40 \\
\hline \multicolumn{5}{|c|}{ Glyphosate Potassium (mM) } \\
\hline 25 & 76.2 & $1.5 \pm 0.2$ & $5.5 \pm 0.1$ & 90.3 \\
\hline 50 & 87.4 & $4.7 \pm 0.2$ & $2.7 \pm 0.1$ & 66.5 \\
\hline 100 & 87.5 & $3.6 \pm 0.3$ & $3.5 \pm 0.2$ & 37.3 \\
\hline 200 & 93 & $2.8 \pm 0.1$ & $4.7 \pm 0.1$ & 36.5 \\
\hline \multicolumn{5}{|c|}{ Tebuconazole (mM) } \\
\hline 25 & 66.4 & $1.5 \pm 0.1$ & $4.9 \pm 0.1$ & 53.8 \\
\hline 50 & 66.5 & $4.9 \pm 0.2$ & $3.1 \pm 0.1$ & 45.3 \\
\hline 100 & 73.4 & $3.7 \pm 3.5$ & $3.8 \pm 0.2$ & 37.6 \\
\hline 200 & 90.9 & $2.6 \pm 0.2$ & $4.4 \pm 0.2$ & 36.9 \\
\hline \multicolumn{5}{|c|}{ Chlorpyrifos-methyl (mM) } \\
\hline 25 & 61.5 & $4.6 \pm 0.1$ & $2.8 \pm 0.2$ & 81.1 \\
\hline 50 & 70.6 & $4.2 \pm 0.3$ & $2.9 \pm 0.2$ & 93.8 \\
\hline 100 & 93 & $4.1 \pm 0.3$ & $3.1 \pm 0.1$ & 35.7 \\
\hline 200 & 170.1 & $3.7 \pm 0.4$ & $3.5 \pm 0.1$ & 33.8 \\
\hline \multicolumn{5}{|c|}{ Deltamethrin (mM) } \\
\hline 2.5 & 60.1 & $1.8 \pm 0.1$ & $5.4 \pm 0.1$ & 59.2 \\
\hline 5 & 64.3 & $3.8 \pm 0.1$ & $3.4 \pm 0.1$ & 48.8 \\
\hline 10 & 93.7 & $1.9 \pm 0.1$ & $4.7 \pm 0.1$ & 41.9 \\
\hline 20 & 100 & $1.5 \pm 0.1$ & $4.4 \pm 0.2$ & 37.7 \\
\hline Mix 50 & 66.4 & $1.4 \pm 0.1$ & $5.9 \pm 0.1$ & 33.8 \\
\hline Mix 200 & 59.4 & $1.3 \pm 0.1$ & $6.1 \pm 0.1$ & 25.3 \\
\hline
\end{tabular}

which trigger important risk factors for public health. Pesticide exposure has different acute and chronic effects, including cancer, asthma, diabetes, and neurodegeneration (Kim, Kabir \& Jahan, 2017). One of the main mechanisms underlying reasons of pesticide toxicity is oxidative stress. Pesticides have induced oxidative stress mechanisms in relation with diseases, and this is still a question of debate. Oxidative stress is a cellular homeostatic imbalance between ROS and antioxidants. While ROS increases in the cell, ROS products interact with important cellular molecules and cause detrimental effects in the cell that bring about several different diseases. Antioxidants are very important cellular soldiers against ROS products. There are several different studies on the oxidative stress induction effects of pesticides in the literature (Agrawal \&Sharma, 2010).

In our study, we have investigated the cytotoxicity and oxidative stress inducing effects of six different pesticides (chlorpyrifos-methyl, pirimiphos-methyl, glyphosate, chlormequat chloride, deltamethrin, and tebuconazole) and the exposure of the mixture of them on the HUVEC cell line. HUVEC are very important for analyzing endothelial markers and representing vascular physiology in vitro. It is a general model for endothelial cells with normal and pathological condition analyses. The HUVEC cell line is a good choice for analyzing xenobiotic toxic effects (Cao et al. 2017; Benachour \& Séralini, 2009). 
It has been reported that LDH levels increase during oxidative stress induction in the cells (Jovanovic et al., 2010). According to our results, LDH levels decrease in a lower concentration (25 $\mathrm{mM}$ ) in all pesticide groups. LDH levels significantly decrease in all chlormequat chloride concentrations. Increased LDH levels are observed in a higher concentration of chlorpyrifos-methyl and pirimiphos-methyl groups. The increased LDH level of higher concentrations is associated with why these concentrations have exceeded $I C_{50}$ values. Decreased LDH levels are observed significantly in 50 and $200 \mathrm{mM}$ mix groups compared to the control group. In this study, TAS levels decrease and TOS levels increase in all groups, and this is balanced in itself. This is the first study to demonstrate chlormequat cloride effects on LDH levels and oxidative stress. It has been demonstrated that chlorpyrifos-methyl, pirimiphos-methyl, glyphosate, chlormequat chloride, deltamethrin, and tebuconazole increased LDH and oxidative stress levels in different studies (Banaee et al. 2019; Hatami, Banaee, Nematdoodt, \& Haghi 2019; Nasr, El-Demerdash \& El-Nagar, 2016; Yıldırım et al. 2013; Sai et al. 2014; Tilak, Veeraiah \& Rao, 2005; Mansour \& Mossa, 2011; Piatti, Marabini \& Chiesara, 1994; Martínez et al 2020; Li et al. 2017; Gholami-Seyedkolaei, Mirvaghefi, Farahmand, \& Kosari 2013; Gündüz et al. 2015; Abdel-Daim et al. 2016; Ncir et al. 2016; Berrouague et al. 2019; Ben Othmène et al.2020), and our result is in accordance with previous studies.

In our study, GSH levels significantly decreased concentration dependent for all exposure concentrations in the chlormequat chloride, deltamethrin, tebuconazole, and mixture groups. In the glyphosate group of 50, 100 and 200 mM concentrations, GSH levels decreased significantly compared to the control group. In pirimiphos-methyl 100 and 200 mM concentrations, GSH levels decreased significantly compared to the control group. For chlorpyrifos-methyl group 100 and 200 mM concentrations, GSH levels decreased significantly compared to the control group. This is the first study showing chlormequat chloride and Tebuconazole's effects on GSH level. This study is done in accordance with previous studies on decreasing GSH in the literature for deltamethrin (Zaki, Algaleel, Imam, Soliman \& Ghoneim, 2020; Bhattacharjee, Borah \& Das, 2020; Kumar, Sharma, Rana \& Sharma, 2019; Yang et al. 2020; Ndonwi et al. 2019; Wang, Shen, Zhou, \& Jin 2019; Velki \&Hackenberger, 2013).

In conclusion, living organisms always encounter different types of pesticides and their combination in daily life through different exposure pathways. It is very important to clarify their toxic effect mechanisms and relations with diseases to make effective risk assessments and set regulations based on this information. Endothelial cells situate in the first phase of exposure to xenobiotics, and they are affected more. This study has confirmed that the oxidative stress inducing effects of exposure to different types of pesticides and their mixture (chlorpyrifos-methyl, pirimiphos-methyl, glyphosate, chlormequat chloride, deltamethrin, tand tebuconazole) and reducing antioxidant capacity of the cells. Further in vitro and in vivo studies need to clarify molecular interactions between pesticide inducing oxidative stress and endothelial cell related diseases. When these mechanisms are understood, protective and therapeutic strategies for the treatment of pesticide exposure's toxic effects can be developed.

Peer-review: Externally peer-reviewed.

Author contributions: Conception/Design of Study- Ç.S.; Data Acquisition- A.T.; Data Analysis/Interpretation-M.K.; Drafting ManuscriptM.K.; Critical Revision of Manuscript-Ç.S.; Final Approval and Accountability- Ç.S., A.T., M.K.

Conflict of Interest: The authors have no conflict of interest to declare.

Financial Disclosure: This study was supported by Ataturk University Scientific Research Projects Fund (Project Number:THD-2018-6548).

\section{REFERENCES}

- $\quad$ Abdel-Daim, M., El-Bialy, B. E., Rahman, H. G., Radi, A. M., Hefny, H. A., \& Hassan, A. M. (2016). Antagonistic effects of Spirulina platensis against sub-acute deltamethrin toxicity in mice: Biochemical and histopathological studies. Biomedicine \& Pharmacotherapy= Biomedecine \& Pharmacotherapie, 77, 79-85.

Abdollahi, M., Ranjbar, A., Shadnia, S., Nikfar, S., \& Rezaie, A. (2004). Pesticides and oxidative stress: a review. Medical Science Monitor: International Medical Journal of Experimental and Clinical Research, 10(6), RA141-RA147.

- $\quad$ Agrawal, A., \& Sharma, B., (2010). Pesticides induced oxidative stress in mammalian systems. International Journal of Biological and Medical Research, 1(3), 90-104.

- Anogwih, J. A. (2014). Toxicity of pirimiphos methyl (Actellic 25EC) on Anopheles gambiae s.s., Culex quinquefasciatus (Diptera: Culicidae), and potential biocontrol agent, Poecilia reticulata (Pisces: Poeciliidae). Journal of Economic Entomology, 107(4), 1440-1446.

- Bagherpour Shamloo, H., Golkari, S., Faghfoori, Z., Movassaghpour, A., Lotfi, H., Barzegari, A., \& Yari Khosroushahi, A. (2016). Lactobacillus Casei Decreases Organophosphorus Pesticide Diazinon Cytotoxicity in Human HUVEC Cell Line. Advanced Pharmaceutical Bulletin, 6(2), 201-210.

- Banaee, M., Akhlaghi, M., Soltanian, S., Gholamhosseini, A., Heidarieh, H., \& Fereidouni, M. S. (2019). "Acute exposure to chlorpyrifos and glyphosate induces changes in hemolymph biochemical parameters in the crayfish, Astacus leptodactylus (Eschscholtz, 1823)". Comparative Biochemistry and Physiology. Toxicology \& Pharmacology, 222, 145-155.

- Ben Othmène, Y., Hamdi, H., Annabi, E., Amara, I., Ben Salem, I., Neffati, F., Najjar, M. F., Abid-Essefi, S. (2020). Tebuconazole induced cardiotoxicity in male adult rat. Food and chemical toxicology An International Journal Published for the British Industrial Biological Research Association, 137, 111134.

- Benachour, N., \& Séralini, G. E. (2009). Glyphosate formulations induce apoptosis and necrosis in human umbilical, embryonic, and placental cells. Chemical Research in Toxicology, 22(1), 97-105. Berrouague, S., Rouag, M., Khaldi, T., Boumendjel, A., Boumendjel, M., Taibi, F., \& Messarah, M. (2019). Efficacy of Allium sativum oil to alleviate tebuconazol-induced oxidative stress in the liver of adult rats. Cellular and Molecular Biology (Noisy-le-Grand, France), 65(8), 23-31.

- Bhattacharjee, P., Borah, A., \& Das, S. (2020). Quercetin-induced amelioration of Deltamethrin stress in freshwater teleost, Channa punctata: Multiple biomarker analysis. Comparative Biochemistry and Physiology. Toxicology \& Pharmacology, 227, 108626.

- Cai, W., Yang, X., Li, X., Li, H., Wang, S., Wu, Z., Yu, M., Ma, S., \& Tang, S. (2020). Low-dose Roundup induces developmental toxicity in bovine preimplantation embryos in vitro. Environmental Science and Pollution Research International, 27(14), 16451-16459. 
- Cao, Y., Gong, Y., Liu, L., Zhou, Y., Fang, X., Zhang, C., Li, Y., \& Li, J. (2017). The use of human umbilical vein endothelial cells (HUVECs) as an in vitro model to assess the toxicity of nanoparticles to endothelium: a review. Journal of Applied Toxicology, 37(12), 1359-1369.

- Deng, Y., Zhang, Y., Lu, Y., Zhao, Y., \& Ren, H. (2016). Hepatotoxicity and nephrotoxicity induced by the chlorpyrifos and chlorpyrifosmethyl metabolite, 3,5,6-trichloro-2-pyridinol, in orally exposed mice". The Science of the Total Environment, 544, 507-514.

- Dokuyucu, R., Bilgili, A., Hanedan, B., Dogan, H., Dokuyucu, A., \& Celik, M. M. (2016). Attenuating effects of caffeic acid phenethyl ester with intralipid on hepatotoxicity of chlorpyrifos in the case of rats. Medicina, 67(6), 743-749.

- $\quad$ Ferreira, D., da Motta, A. C., Kreutz, L. C., Toni, C., Loro, V. L., \& BarcelIos, L. J. (2010). Assessment of oxidative stress in Rhamdia quelen exposed to agrichemicals. Chemosphere, 79(9), 914-921.

- Gholami-Seyedkolaei, S. J., Mirvaghefi, A., Farahmand, H., \& Kosari, A. A. (2013). Effect of a glyphosate-based herbicide in Cyprinus carpio: assessment of acetylcholinesterase activity, hematological responses and serum biochemical parameters. Ecotoxicology and Environmental Safety, 98, 135-141.

- Gündüz, E., Ülger, B. V., İbiloğlu, I., Ekinci, A., Dursun, R., Zengin, Y., İçer, M., Uslukaya, Ö., Ekinci, C., \& Güloğlu, C. (2015). Glutamine provides effective protection against deltamethrin-induced acute hepatotoxicity in rats but not against nephrotoxicity. Medical Science Monitor:International Medical Journal of Experimental and Clinical Research, 21, 1107-1114.

- Hatami, M., Banaee, M., \& Nematdoost Haghi, B. (2019). Sub-lethal toxicity of chlorpyrifos alone and in combination with polyethylene glycol to common carp (Cyprinus carpio). Chemosphere, 219, 981-988.

- Jovanovic, P., Zoric, L., Stefanovic, I., Dzunic, B., Djordjevic-Jocic, J., Radenkovic, M., \& Jovanovic, M. (2010). Lactate dehydrogenase and oxidative stress activity in primary open-angle glaucoma aqueous humour. Bosnian Journal of Basic Medical Sciences, 10(1), 83-88.

- Kim, K. H., Kabir, E., \& Jahan, S. A. (2017). Exposure to pesticides and the associated human health effects. The Science of the Total Environment, 575, 525-535.

- Kumar, A., Sharma, R., Rana, D., \& Sharma, N. (2019). Protective Effect of Alpha-Tocopherol in Deltamethrin Induced Immunotoxicity, Endocrine, Metabolic \& Immune Disorders - Drug Targets, 19(2), 171-184.

- Laetz, C. A., Baldwin, D. H., Collier, T. K., Hebert, V., Stark, J. D., \& Scholz, N. L. (2009). The synergistic toxicity of pesticide mixtures: implications for risk assessment and the conservation of endangered Pacific salmon. Environmental Health Perspectives, 117(3), 348-353.

- $\quad$ Li, M. H., Ruan, L. Y., Zhou, J. W., Fu, Y. H., Jiang, L., Zhao, H., \& Wang, J. S. (2017). Metabolic profiling of goldfish (Carassius auratis) after long-term glyphosate-based herbicide exposure. Aquatic Toxicology (Amsterdam, Netherlands), 188, 159-169.

- $\quad$ Li, S., Jiang, Y., Sun, Q., Coffin, S., Chen, L., Qiao, K., Gui, W., \& Zhu, G. (2020). Tebuconazole induced oxidative stress related hepatotoxicity in adult and larval zebrafish (Danio rerio). Chemosphere, 241, 125129.

- Lu, Q., Sun, Y., Ares, l., Anadón, A., Martínez, M., Martínez-Larrañaga, M. R., Yuan, Z., Wang, X., \& Martínez, M. A. (2019). Deltamethrin toxicity: A review of oxidative stress and metabolism. Environmental Research, 170, 260-281.

- Mansour, S. A., \& Mossa, A. T. (2011). Adverse effects of exposure to low doses of chlorpyrifos in lactating rats. Toxicology and Industrial Health, 27(3), 213-224.

- Martínez, M. A., Rodríguez, J. L., Lopez-Torres, B., Martínez, M., Martínez-Larrañaga, M. R., Maximiliano, J. E., Anadón, A., \& Ares, I. (2020). Use of human neuroblastoma SH-SY5Y cells to evaluate glyphosate-induced effects on oxidative stress, neuronal development and cell death signaling pathways. Environment International, 135, 105414.
Medina-Leyte, D. J., Domínguez-Pérez, M., Mercado, I., VillarrealMolina, M.T., \& Jacobo-Albavera, L. (2020). Use of human umbilical vein endothelial cells (HUVEC) as a model to study cardiovascular disease: A review. Applied Sciences, 10(3), 938.

Nasr, H. M., El-Demerdash, F. M., \& El-Nagar, W. A. (2016). Neuro and renal toxicity induced by chlorpyrifos and abamectin in rats: Toxicity of insecticide mixture. Environmental Science and Pollution Research International, 23(2), 1852-1859.

Ncir, M., Ben Salah, G., Kamoun, H., Makni Ayadi, F., Khabir, A., El Feki, A., \& Saoudi, M. (2016). Histopathological, oxidative damage, biochemical, and genotoxicity alterations in hepatic rats exposed to deltamethrin: modulatory effects of garlic (Allium sativum). Canadian journal of Physiology and Pharmacology, 94(6), 571-578. Ndonwi, E. N., Atogho-Tiedeu, B., Lontchi-Yimagou, E., Shinkafi, T. S., Nanfa, D., Balti, E. V., Indusmita, R., Mahmood, A., Katte, J. C., Mbanya, A., Matsha, T., Mbanya, J. C., Shakir, \& A., Sobngwi, E. (2019). Gestational Exposure to Pesticides Induces Oxidative Stress and Lipid Peroxidation in Offspring that Persist at Adult Age in an Animal Model. Toxicological Research, 35(3), 241-248.

- $\quad$ Odetti, L. M., López González, E. C., Romito, M. L., Simoniello, M. F., \& Poletta, G. L. (2020). Genotoxicity and oxidative stress in Caiman latirostris hatchlings exposed to pesticide formulations and their mixtures during incubation period. Ecotoxicology and Environmental Safety, 193, 110312

- $\quad$ Olsvik, P. A., Berntssen, M., \& Søfteland, L. (2017). In vitro toxicity of pirimiphos-methyl in Atlantic salmon hepatocytes. Toxicology in Vitro, 39, 1-14.

Oyesola, T., Iranloye, B., \& Adegoke, O. (2019). Implantation and pregnancy outcome of Sprague-Dawley rats exposed to pirimiphos-methyl. Endocrine Regulations, 53(3), 139-145.

Piatti, E., Marabini, L., \& Chiesara, E. (1994). Increase of micronucleus frequency in cultured rat hepatocytes treated in vitro with benomyl and pirimiphos-methyl separately and in mixture. Mutation Research, 324(1-2), 59-64.

Roelofs, M., Temming, A. R., Piersma, A. H., van den Berg, M., \& van Duursen, M. (2014). Conazole fungicides inhibit Leydig cell testosterone secretion and androgen receptor activation in vitro. Toxicology Reports, 1, 271-283.

- $\quad$ Sai, L., Li, X., Liu, Y., Guo, Q., Xie, L., Yu, G., Bo, C., Zhang, Z., \& Li, L. (2014). Effects of chlorpyrifos on reproductive toxicology of male rats. Environmental Toxicology, 29(9), 1083-1088.

- $\quad$ Staal, Y., Meijer, J., van der Kris, R., de Bruijn, A. C., Boersma, A. Y., Gremmer, E. R., Zwart, E. P., Beekhof, P. K., Slob, W., \& van der Ven, L. (2018). Head skeleton malformations in zebrafish (Danio rerio) to assess adverse effects of mixtures of compounds. Archives of Toxicology, 92(12), 3549-3564

- Tilak, K. S., Veeraiah, K., \& Rao, D. K. (2005). Biochemical changes induced by chlorpyrifos, an organophosphate compound in sublethal concentrations to the freshwater fish Catla catla, Labeo rohita and Cirrhinus mrigala. Journal of Environmental Biology, $26(2$ Suppl), 341-347.

- Velki, M., \& Hackenberger, B. K. (2013). Different sensitivities of biomarker responses in two epigeic earthworm species after exposure to pyrethroid and organophosphate insecticides. Archives of Environmental Contamination and Toxicology, 65(3), 498-509.

Vijitharan, V., Warnasekare, J., Lokunarangoda, N. C., Farah, M. F., \& Siribaddana, S. H. (2016). Fatal poisoning with plant growth regulator - chlormequat. The Ceylon Medical Journal, 61 (2), 89-90.

Wang, X., Shen, M., Zhou, J., \& Jin, Y. (2019). Chlorpyrifos disturbs hepatic metabolism associated with oxidative stress and gut microbiota dysbiosis in adult zebrafish. Comparative Biochemistry and Physiology. Toxicology \& Pharmacology, 216, 19-28.

Xiagedeer, B., Wu, S., Liu, Y., \& Hao, W. (2016). Chlormequat chloride retards rat embryo growth in vitro. Toxicology in Vitro, 34, 274-282. 
Istanbul J Pharm 51 (2): 183-190

- Yang, J., Gong, Y., Cai, J., Zheng, Y., Liu, H., \& Zhang, Z. (2020). "Chlorpyrifos induces redox imbalance-dependent inflammation in common carp lymphocyte through dysfunction of T-cell receptor $\gamma^{\prime \prime}$. Journal of Fish Diseases, 43(4), 423-430.

- Yıldırım, E., Baydan, E., Kanbur, M., Kul, O., Cınar, M., Ekici, H., \& Atmaca, N. (2013). "The effect of chlorpyrifos on isolated thoracic aorta in rats." BioMed Research International, 2013, 376051.
- Zaki, S. M., Algaleel, W., Imam, R. A., Soliman, G. F., \& Ghoneim, F. M. (2020). Nano-curcumin versus curcumin in amelioration of deltamethrin-induced hippocampal damage. Histochemistry and Cell Biology, 154(2), 157-175

Zhang, H. C., Yang, Y. J., Ma, K. X., Shi, C. Y., Chen, G. W., \& Liu, D. Z. (2020). "A novel sigma class glutathione S-transferase gene in freshwater planarian Dugesia japonica: cloning, characterization and protective effects in herbicide glyphosate stress". Ecotoxicology (London, England), 29(3), 295-304. 